\title{
The Pd(0) nanoparticles stabilized by collagen fibres as a recyclable heterogeneous catalyst for the Stille reaction under aerobic condition
}

\author{
BABAK MOHAMMADI-AGHDAM ${ }^{\mathrm{a}, *}$, SIAVASH BAHARI ${ }^{\mathrm{b}}$ and RAHIM MOLAEI ${ }^{\mathrm{a}}$ \\ ${ }^{a}$ Faculty of Science, Urmia Branch, Islamic Azad University, Urmia 57146, Iran \\ ${ }^{b}$ Department of Chemistry, Ahar Branch, Islamic Azad University, Ahar 54517, Iran \\ e-mail: b.mohamadi@yahoo.com
}

MS received 29 April 2012; revised 30 October 2012; accepted 26 November 2012

\begin{abstract}
The stabilized palladium(0) nanoparticles by collagen fibres was a highly active, air-stable and recyclable heterogeneous catalyst that could be used for the Stille coupling reactions between aryl iodides and organostannanes under aerobic conditions. This method offered the several advantages: high yield under facile reaction condition and easy work-up procedure. The catalyst was easily recovered from the reaction mixture by filtration and reused multiple times without significant reduction or decrease in the activity.
\end{abstract}

Keywords. Stille coupling; $\operatorname{Pd}(0)$ nanoparticle catalyst; organostannanes; aryl iodide; heterogeneous catalyst; collagen fibres.

\section{Introduction}

The Stille cross-coupling reactions of organohalides with organotin compounds have been proven to be a useful synthetic method for the $\mathrm{C}-\mathrm{C}$ bond formation in organic synthesis. Many homogeneous palladium catalysts such as $\mathrm{Pd}\left(\mathrm{PPh}_{3}\right)_{4}, \mathrm{PdCl}_{2}\left(\mathrm{PPh}_{3}\right)_{2}$ and $\mathrm{PdCl}_{2}(\mathrm{MeCN})_{2}$ for the Stille cross-coupling reaction are known. ${ }^{1-5}$ Unfortunately, all of these known methods generally required dry, oxygen-free and drastic reaction conditions and tedious work-up procedures due to use of homogeneous catalyst. Therefore, it is desirable to develop a more efficient and convenient method employing the heterogeneous catalytic conditions. ${ }^{6}$

In recent years, heterogeneous catalysts have played a crucial role in organic syntheses because of economic and environmental considerations. These catalysts are generally less expensive, eco-friendly, highly reactive, easy to handle and recoverable. ${ }^{7}$

A number of effective methods have been reported in recent years for the Stille coupling reactions in the presence of heterogeneous catalysts. ${ }^{\text {6a }}$ These catalysts possessing high activity have attracted significant interest because they can be easily recovered and reused, leading to a reduction in waste. The development of a catalytic synthetic method for still reaction remains an active research area.

Collagen is a class of renewable biopolymers originated from the skin of domestic animals, which are

*For correspondence widely used as a potential biomaterial for the preparation of catalyst supporter. Collagen fibre-supported Pd catalysts having high activity and selectivity are currently attracting a great interest due to their easy separation and recovery. ${ }^{8}$

However, to the best of our knowledge, Stille reactions catalysed by CF-supported Pd catalysts have received less attention. This encouraged us to investigate the $\operatorname{Pd}(0)$ nanoparticle catalyst stabilized by EGCG-grafted CF (Pd(0)-EGCG-CF) ${ }^{8 e}$ for the Stille reaction (scheme 1). Our approach was guided by three imperatives: (i) the biopolymer reagent should be easily accessible; (ii) starting from readily available and cheap reagents; and (iii) the biopolymer reagent should be air stable, which should allow its storage in normal bottles with extended shelf life.

We report here a new protocol for the Stille coupling of aryl iodides with organostannanes under aerobic conditions using $\mathrm{Pd}(0)$ nanoparticle catalyst stabilized by EGCG-grafted CF (Pd(0)-EGCG-CF) as an efficient heterogeneous catalyst (scheme 1). This catalyst is safe, easy to handle, air stable, environmentally benign with fewer disposals problems. The catalyst was characterized by using powder XRD, XPS, SEM, TEM and FT-IR spectroscopy. ${ }^{8 \mathrm{e}}$

\section{Experimental}

\subsection{General}

All reagents were purchased from Merck and Aldrich and used without further purification. Products were 


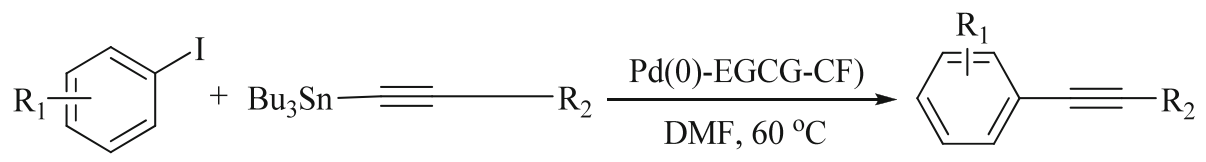

Scheme 1. Stille coupling of aryl iodides with alkynylstannanes in the presence of Pd(0)-EGCG-CF.

characterized by spectroscopy data (NMR spectra), and melting points. The NMR spectra were recorded in DMSO and $\mathrm{CDCl}_{3} \cdot{ }^{1} \mathrm{H}$ NMR spectra were recorded on a Bruker Avance DRX 300 and $400 \mathrm{MHz}$ instruments.

\subsection{General procedure for the Stille coupling reaction}

Aryl iodide (1.0 mmol), Pd(0)-EGCG-CF (2.0 mol\%), and DMF $(3 \mathrm{~mL})$ were added to a flask, and the resulting mixture was stirred at room temperature for $15 \mathrm{~min}$ under aerobic conditions. To this, suspension was added organostannane $(1.2 \mathrm{mmol})$ and the reaction mixture was stirred at $60^{\circ} \mathrm{C}$ for an appropriate time (TLC). The mixture was dissolved in $\mathrm{Et}_{2} \mathrm{O}(30 \mathrm{~mL})$. The $\mathrm{Pd}(0)-$ EGCG-CF catalyst was separated from the reaction mixture by filtration after each experiment, washed with water and acetonitrile and dried carefully before use in subsequent runs. The ethereal solution was treated with $20 \%$ aqueous $\mathrm{KF}(10 \mathrm{~mL})$ for $30 \mathrm{~min}$ before being dried and concentrated. The solvent was removed under vacuum and the residue was purified by flash chromatography on silica gel using ethyl acetate/hexane as eluent to give the desired product. The pure products were characterized by IR, ${ }^{1} \mathrm{H}$ NMR and melting point. All the products are known compounds and the spectral data and melting points were identical to those reported in the literature. ${ }^{9}$

\subsection{Spectroscopic data of selected products}

2.3a Diphenylacetylene (table 2, entry 1): Mp 61$62{ }^{\circ} \mathrm{C}$ (lit. $\left.{ }^{17} 60-62{ }^{\circ} \mathrm{C}\right) ;{ }^{1} \mathrm{H}$ NMR $\left(\mathrm{CDCl}_{3}, 300 \mathrm{MHz}\right) \delta$ 7.55-7.50 (m, 4H), 7.34-7.30 (m, 6H).

$2.3 \mathrm{~b}$ (4-Methoxyphenyl)phenylacetylene (table 2, entry 2): $\mathrm{Mp} 60-61^{\circ} \mathrm{C}$ (lit. ${ }^{18} 57-61^{\circ} \mathrm{C}$ ); ${ }^{1} \mathrm{H}$ NMR $\left(\mathrm{CDCl}_{3}, 300 \mathrm{MHz}\right) \delta 7.57-7.51(\mathrm{~m}, 2 \mathrm{H}), 7.50(\mathrm{~d}$, $J=8.9 \mathrm{~Hz}, 2 \mathrm{H}), 7.40-7.35(\mathrm{~m}, 3 \mathrm{H}), 6.91(\mathrm{~d}$, $J=8.9 \mathrm{~Hz}, 2 \mathrm{H}), 3.86(\mathrm{~s}, 3 \mathrm{H})$.

2.3c Phenyl-p-tolyacetylene (table 2, entry 3): $\mathrm{Mp}$ 70-72 ${ }^{\circ} \mathrm{C}$ (lit. $\left.{ }^{19} 72-73^{\circ} \mathrm{C}\right) ;{ }^{1} \mathrm{H}$ NMR $\left(\mathrm{CDCl}_{3}, 300 \mathrm{MHz}\right)$ $\delta 7.53-7.48(\mathrm{~m}, 2 \mathrm{H}), 7.43(\mathrm{~d}, J=7.91 \mathrm{~Hz}, 2 \mathrm{H})$, $7.30-7.25(\mathrm{~m}, 3 \mathrm{H}), 7.10(\mathrm{~d}, J=7.91 \mathrm{~Hz}, 2 \mathrm{H})$, $2.32(\mathrm{~s}, 3 \mathrm{H})$.

2.3d (4-Acetylphenyl)phenylacetylene (table 2, entry 4): $\mathrm{Mp} 95-97^{\circ} \mathrm{C}$ (lit. ${ }^{20} 94-96^{\circ} \mathrm{C}$ ); ${ }^{1} \mathrm{H}$ NMR $\left(\mathrm{CDCl}_{3}, 300 \mathrm{MHz}\right) \delta 7.94(\mathrm{~d}, J=8.52 \mathrm{~Hz}, 2 \mathrm{H}), 7.60$ $(\mathrm{d}, J=8.52 \mathrm{~Hz}, 2 \mathrm{H}), 7.55-7.51(\mathrm{~m}, 2 \mathrm{H}), 7.39-7.35$ (m, 3H), 2.61 (s, 3H).

$2.3 \mathrm{e}$ (4-Nitrophenyl)phenylacetylene (table 2, entry 5): $\mathrm{Mp} 121-122^{\circ} \mathrm{C}$ (lit. $\left.{ }^{21} 120-121^{\circ} \mathrm{C}\right) ;{ }^{1} \mathrm{H}$ NMR $\left(\mathrm{CDCl}_{3}, 300 \mathrm{MHz}\right) \delta 8.21(\mathrm{~d}, J=8.83 \mathrm{~Hz}, 2 \mathrm{H}), 7.66$ $(\mathrm{d}, J=8.73 \mathrm{~Hz}, 2 \mathrm{H}), 7.57-7.53(\mathrm{~m}, 2 \mathrm{H}), 7.40-7.37$ (m, 3H).

$2.3 \mathrm{f}$ (3-Nitrophenyl)phenylacetylene (table 2, entry 6): $\mathrm{Mp} 68-70^{\circ} \mathrm{C}$ (lit. ${ }^{22} 68-70^{\circ} \mathrm{C}$ ); ${ }^{1} \mathrm{H}$ NMR $\left(\mathrm{CDCl}_{3}, 300 \mathrm{MHz}\right) \delta 8.32(\mathrm{~s}, 1 \mathrm{H}), 8.12(\mathrm{~d}, J=8.10 \mathrm{~Hz}$, $1 \mathrm{H}), 7.77(\mathrm{~d}, J=7.81 \mathrm{~Hz}, 1 \mathrm{H}), 7.52-7.48(\mathrm{~m}, 3 \mathrm{H})$, 7.36-7.31 (m, 3H).

2.3g 1-Phenylhex-1-yne (table 2, entry 7): $\quad \mathrm{Oil}^{23} ;{ }^{1} \mathrm{H}$ NMR $\left(\mathrm{CDCl}_{3}, 300 \mathrm{MHz}\right) \delta 0.97(\mathrm{t}, J=7.4 \mathrm{~Hz}, 3 \mathrm{H})$, $1.65-1.49(\mathrm{~m}, 4 \mathrm{H}), 2.43$ (t, $J=7.4 \mathrm{~Hz}, 2 \mathrm{H}), 7.30-7.25$ (m, 3H), 7.44-7.41 (m, 2H).

2.3h 1-(1-Hexynyl)-4-methylbenzene (table 2, entry 8): $\mathrm{Oil}^{24} ;{ }^{1} \mathrm{H} \mathrm{NMR}\left(\mathrm{CDCl}_{3}, 400 \mathrm{MHz}\right) \delta 0.92(\mathrm{t}$, $J=7.4 \mathrm{~Hz}, 3 \mathrm{H}), 1.61-1.42(\mathrm{~m}, 4 \mathrm{H}), 2.30$ (s, 3H), 2.37 $(\mathrm{t}, J=7.0 \mathrm{~Hz}, 2 \mathrm{H}), 7.05(\mathrm{~d}, J=8.5 \mathrm{~Hz}, 2 \mathrm{H}), 7.25(\mathrm{~d}$, $J=8.5 \mathrm{~Hz}, 2 \mathrm{H})$.

2.3i 1-(1-Hexynyl)-4-nitrobenzene (table 2, entry 9): $\mathrm{Oil}^{14} ;{ }^{1} \mathrm{H}$ NMR $\left(\mathrm{CDCl}_{3}, 400 \mathrm{MHz}\right) \delta 8.17(\mathrm{~d}, J=$ $8.9 \mathrm{~Hz}, 2 \mathrm{H}), 7.53(\mathrm{~d}, J=8.9 \mathrm{~Hz}, 2 \mathrm{H}), 2.50(\mathrm{t}, J=$ $7.1 \mathrm{~Hz}, 2 \mathrm{H}), 1.65-1.58(\mathrm{~m}, 2 \mathrm{H}), 1.53-1.48(\mathrm{~m}, 2 \mathrm{H})$, $0.98(\mathrm{t}, J=7.1 \mathrm{~Hz}, 3 \mathrm{H})$.

2.3j 1-(1-Hexynyl)-4-methoxybenzene (table 2, entry 10): $\mathrm{Oil}^{25} ;{ }^{1} \mathrm{H} \mathrm{NMR}\left(\mathrm{CDCl}_{3}, 300 \mathrm{MHz}\right) \delta 0.97(\mathrm{t}, J=$ $7.4 \mathrm{~Hz}, 3 \mathrm{H}), 1.64-1.45(\mathrm{~m}, 4 \mathrm{H}), 2.91(\mathrm{t}, J=7.1 \mathrm{~Hz}$, $2 \mathrm{H}), 3.82(\mathrm{~s}, 3 \mathrm{H}), 6.8 \mathrm{k}(\mathrm{d}, J=8.8 \mathrm{~Hz}, 2 \mathrm{H}), 7.34(\mathrm{~d}$, $J=8.8 \mathrm{~Hz}, 2 \mathrm{H})$. 
2.3k Trimethyl(2-phenyl-1-ethynyl)silane (table 2, entry 11): $\mathrm{Oil}^{26} ;{ }^{1} \mathrm{H}$ NMR $\left(\mathrm{CDCl}_{3}, 300 \mathrm{MHz}\right) \delta$ 7.47-7.43 (m, 2H), 7.31-7.28 (m, 3H), $0.24(\mathrm{~m}$, $9 \mathrm{H})$.

2.31 Trimethyl[(4-nitrophenyl)ethynyl]silane (table 2, entry 12): $\mathrm{Oil}^{10} ;{ }^{1} \mathrm{H}$ NMR $\left(\mathrm{CDCl}_{3}, 300 \mathrm{MHz}\right) \delta 8.16$ $(\mathrm{d}, J=8.7 \mathrm{~Hz}, 2 \mathrm{H}), 7.61(\mathrm{~d}, J=8.7 \mathrm{~Hz}, 2 \mathrm{H}), 0.18(\mathrm{~s}$, 9H).

2.3m (E) 1-(3-methoxyprop-1-enyl)benzene (table 3, entry 1): $\mathrm{Oil}^{16} ;{ }^{1} \mathrm{H} \mathrm{NMR}\left(\mathrm{CDCl}_{3}, 300 \mathrm{MHz}\right) \delta 3.37$ (s, $3 \mathrm{H}), 4.04(\mathrm{~d}, J=6.1 \mathrm{~Hz}, 2 \mathrm{H}), 6.27-6.30(\mathrm{~m}, 1 \mathrm{H}), 6.62$ $(\mathrm{d}, J=15.9 \mathrm{~Hz}, 1 \mathrm{H}), 7.42-7.20(\mathrm{~m}, 5 \mathrm{H})$.

2.3n (E) 1-methoxy-4-styrylbenzene (table 3, entry 2): $\mathrm{Mp} 135-136^{\circ} \mathrm{C}$ (lit. $\left.{ }^{16} 134-135^{\circ} \mathrm{C}\right) ;{ }^{1} \mathrm{H}$ NMR $\left(\mathrm{CDCl}_{3}\right.$, $400 \mathrm{MHz}) \delta 7.52(\mathrm{~d}, J=7.5 \mathrm{~Hz}, 2 \mathrm{H}), 7.47(\mathrm{~d}, J=$ $8.6 \mathrm{~Hz}, 2 \mathrm{H}), 7.35(\mathrm{t}, J=7.5 \mathrm{~Hz}, 2 \mathrm{H}), 7.27-7.22(\mathrm{~m}$, $1 \mathrm{H}), 7.09(\mathrm{~d}, J=16.7 \mathrm{~Hz}, 1 \mathrm{H}), 7.00(\mathrm{~d}, J=16.7 \mathrm{~Hz}$, $1 \mathrm{H}), 6.92(\mathrm{~d}, J=8.7 \mathrm{~Hz}, 2 \mathrm{H}), 3.85(\mathrm{~s}, 3 \mathrm{H})$.

2.3o (Z) 1-methyl-4-styrylbenzene (table 3, entry 3): $\mathrm{Oil}^{16} ;{ }^{1} \mathrm{H}$ NMR $\left(\mathrm{CDCl}_{3}, 400 \mathrm{MHz}\right) \delta 7.30-7.20(\mathrm{~m}$, $5 \mathrm{H}), 7.17(\mathrm{~d}, J=8.0 \mathrm{~Hz}, 2 \mathrm{H}), 7.06(\mathrm{~d}, J=8.0 \mathrm{~Hz}$, $2 \mathrm{H}), 6.60$ (s, 2H), $2.34(\mathrm{~s}, 3 \mathrm{H})$.

2.3p (E) 1-(hex-1-enyl)-4-methoxybenzene (table 3, entry 4): $\mathrm{Oil}^{16} ;{ }^{1} \mathrm{H} \mathrm{NMR}\left(\mathrm{CDCl}_{3}, 400 \mathrm{MHz}\right) \delta 7.25(\mathrm{~d}$, $J=8.5 \mathrm{~Hz}, 2 \mathrm{H}), 6.82(\mathrm{~d}, J=8.5 \mathrm{~Hz}, 2 \mathrm{H}), 6.29(\mathrm{~d}$, $J=15.4 \mathrm{~Hz}, 1 \mathrm{H}), 5.93(\mathrm{dt}, J=15.4,7.0 \mathrm{~Hz}, 1 \mathrm{H}), 3.82$ (s, 3H), 2.20-2.15 (m, 2H), 1.45-1.33 (m, 4H), $0.91(\mathrm{t}$, $J=7.0 \mathrm{~Hz}, 3 \mathrm{H})$.

2.3q (Z) 1-nitro-4-styrylbenzene (table 3, entry 5): Mp 60-62 ${ }^{\circ} \mathrm{C}$ (lit. $\left.{ }^{16} 60.5-61.5^{\circ} \mathrm{C}\right) ;{ }^{1} \mathrm{H}$ NMR $\left(\mathrm{CDCl}_{3}\right.$, $400 \mathrm{MHz}) \delta 8.10(\mathrm{~d}, J=8.7 \mathrm{~Hz}, 2 \mathrm{H}), 7.40(\mathrm{~d}, J=$ $8.7 \mathrm{~Hz}, 2 \mathrm{H}), 7.25-7.18(\mathrm{~m}, 5 \mathrm{H}), 6.80(\mathrm{~d}, J=12.5 \mathrm{~Hz}$, $1 \mathrm{H}), 6.60(\mathrm{~d}, J=12.5 \mathrm{~Hz}, 1 \mathrm{H})$.

2.3r (Z) 1-(hex-1-enyl)-4-nitrobenzene (table 3, entry 6): $\mathrm{Oil}^{16} ;{ }^{1} \mathrm{H} \mathrm{NMR}\left(\mathrm{CDCl}_{3}, 400 \mathrm{MHz}\right) \delta 8.20(\mathrm{~d}, J=$ $8.6 \mathrm{~Hz}, 2 \mathrm{H}), 7.40(\mathrm{~d}, J=8.6 \mathrm{~Hz}, 2 \mathrm{H}), 6.44(\mathrm{~d}, J=$ $11.5 \mathrm{~Hz}, 1 \mathrm{H}), 5.84(\mathrm{dt}, J=11.5,7.0 \mathrm{~Hz}, 1 \mathrm{H}), 2.35$ $2.30(\mathrm{~m}, 2 \mathrm{H}), 1.49-1.43(\mathrm{~m}, 2 \mathrm{H}), 1.39-1.33(\mathrm{~m}, 2 \mathrm{H})$, $0.89(\mathrm{t}, J=7.0 \mathrm{~Hz}, 3 \mathrm{H})$.

\section{Results and discussion}

In the first set of experiments, for optimization of the reaction conditions, the catalytic potential of $\mathrm{Pd}(0)$ EGCG-CF, the effects of the solvents and temperature was investigated for the reaction between iodobenzene and 1-(tributylstannyl)-2-phenylethyne (table 1). As shown in table 1, the reaction was influenced significantly by the solvent employed. The reaction was successful when DMF and NMP were used as the solvent (entries 4 and 7, table 1), especially resulting in the best result in the case of DMF (entry 7, table 1). THF, $\mathrm{PhH}$ and $\mathrm{CH}_{3} \mathrm{CN}$ were not suitable solvent for this reaction. The optimum amount of $\mathrm{Pd}(0)$-EGCG-CF was found to be $2.0 \mathrm{~mol} \%$ in the presence of iodobenzene (1 mmol) and 1-(tributylstannyl)-2-phenylethyne $(1.2 \mathrm{mmol})$ in DMF $(3 \mathrm{~mL})$ at $60^{\circ} \mathrm{C}$ under the presented reaction condition. The increase in the amount of the palladium catalyst reduced the reaction time whereas it did not improve the yield of the product (entry 10). A low palladium concentration prolonged the reaction time and led to a reduction in the yield (entry 9).

We have examined the coupling reaction of alkynylstannanes with various aryl iodides containing electronwithdrawing or electron-donating groups (scheme 1). As shown in table 2, alkynylstannanes were converted into the corresponding products in high yields under mild conditions.

The optimized catalyst system was quite general and compatible with a wide range of functional groups such as nitro, methoxy, carbonyl and silyl on either partner. We attempted to carry out the coupling reactions of heteroaryl iodides such as 3-iodopyridine with alkynylstannanes under the same reaction conditions,

Table 1. Stille coupling of iodobenzene with 1-(tributylstannyl)-2-phenylethyne under various reaction conditions. ${ }^{\text {a }}$

\begin{tabular}{lccccc}
\hline Entry & Solvent & $\begin{array}{c}\text { Pd(0)-EGCG-CF } \\
(\mathrm{mol} \%)\end{array}$ & $\begin{array}{c}\text { Temp } \\
\left({ }^{\circ} \mathrm{C}\right)\end{array}$ & $\begin{array}{c}\text { Time } \\
(\mathrm{h})\end{array}$ & $\begin{array}{c}\text { Yield }^{\mathrm{b}} \\
(\%)\end{array}$ \\
\hline 1 & THF & 2.0 & 60 & 24 & 20 \\
2 & PhH & 2.0 & 60 & 24 & 21 \\
3 & MeCN & 2.0 & 60 & 24 & 30 \\
4 & NMP & 2.0 & 60 & 5 & 82 \\
5 & DMF & 2.0 & 30 & 24 & 31 \\
6 & DMF & 2.0 & 40 & 24 & 62 \\
7 & DMF & 2.0 & 60 & 5 & 87 \\
8 & DMF & 2.0 & 80 & 5 & 76 \\
9 & DMF & 1.0 & 60 & 9 & 72 \\
10 & DMF & 2.5 & 60 & 5 & 76 \\
\hline
\end{tabular}

a Reaction conditions: iodobenzene $(1 \mathrm{mmol})$, 1(tributylstannyl)-2-phenylethyne (1.2 mmol), solvent ( $3 \mathrm{~mL})$, aerobic conditions

${ }^{\mathrm{b}}$ Isolated yield 
Table 2. Stille coupling of aryl iodides with alkynylstannanes. $^{\text {a }}$

\begin{tabular}{lcccc}
\hline Entry & $\mathrm{R}_{1}$ & $\mathrm{R}_{2}$ & Time (h) & Yield $^{\mathrm{b}}(\%)$ \\
\hline 1 & $\mathrm{H}$ & $\mathrm{Ph}$ & 5 & $88,85^{\mathrm{b}}$ \\
2 & $4-\mathrm{OMe}$ & $\mathrm{Ph}$ & 5 & 86 \\
3 & $4-\mathrm{Me}$ & $\mathrm{Ph}$ & 5 & 84 \\
4 & $4-\mathrm{COMe}$ & $\mathrm{Ph}$ & 5 & 85 \\
5 & $4-\mathrm{NO}_{2}$ & $\mathrm{Ph}$ & 5 & 87 \\
6 & $3-\mathrm{NO}_{2}$ & $\mathrm{Ph}$ & 5 & 86 \\
7 & $\mathrm{H}$ & $\mathrm{n}-\mathrm{C}_{4} \mathrm{H}_{9}$ & 5 & 86 \\
8 & $4-\mathrm{Me}$ & $\mathrm{n}-\mathrm{C}_{4} \mathrm{H}_{9}$ & 7 & 87 \\
9 & $4-\mathrm{NO}_{2}$ & $\mathrm{n}-\mathrm{C}_{4} \mathrm{H}_{9}$ & 5 & 89 \\
10 & $4-\mathrm{OMe}_{1}$ & $\mathrm{n}-\mathrm{C}_{4} \mathrm{H}_{9}$ & 7 & 87 \\
11 & $\mathrm{H}$ & $\mathrm{Me}_{3} \mathrm{Si}$ & 7 & 86 \\
12 & $4-\mathrm{NO}_{2}$ & $\mathrm{Me}_{3} \mathrm{Si}$ & 7 & 85 \\
\hline
\end{tabular}

${ }^{\text {a}}$ Reaction conditions: iodobenzene (1 mmol), alkynylstannanes (1.2 mmol), Pd(0)-EGCG-CF (2.0 mol\%), DMF $(3 \mathrm{~mL})$, aerobic conditions, $65^{\circ} \mathrm{C}$

${ }^{\mathrm{b}}$ Isolated yield

${ }^{\mathrm{c}}$ Yield after the 5 th cycle

but this was unsuccessful, no desired coupled product was detected after $30 \mathrm{~h}$ of reaction time.

The yields of coupled products are high and the organotin reagents can be readily synthesized, purified, and stored. The developed methodology was also applicable for the Stille coupling reactions of vinylstannanes with aryl iodides. The Stille coupling of aryl iodides with a variety of vinylstannanes was investigated (scheme 2), the experimental results are summarized in table 3 . The Stille coupling reactions of a variety of aryl iodides with $(Z)$ - or $(E)$-vinylstannanes proceeded smoothly in the presence of a catalytic amount of $\mathrm{Pd}(0)$-EGCG-CF in DMF at $60^{\circ} \mathrm{C}$ to afford the corresponding coupled products in high yields with retention of the configuration (entries 1-6).

In a typical experiment, after the reaction was completed, $\mathrm{Pd}(0)$-EGCG-CF as a catalyst was isolated from the reaction mixture by simple filtration in the workup stage. The reusability of the catalyst was evaluated after washing the catalyst by water and acetonitrile and drying in an oven. $\mathrm{Pd}(0)$-EGCG-CF was reused for five repeated times with consistent activity (table 2, entry 1 ). Moreover, even after five time uses of the Pd(0)-EGCG$\mathrm{CF}$, there was no decrease in the activity of the catalyst. This reusability demonstrates the high stability and

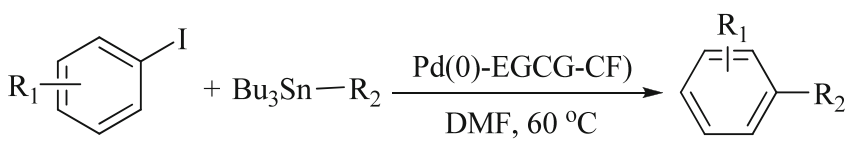

Scheme 2. Stille coupling of aryl iodides with vinylstannanes in the presence of Pd(0)-EGCG-CF.
Table 3. Stille coupling of aryl iodides with vinylstannanes. $^{\text {a }}$

\begin{tabular}{lcccc}
\hline Entry & $\mathrm{R}_{1}$ & $\mathrm{R}_{2}$ & Time (h) & Yield $^{\mathrm{b}}(\%)$ \\
\hline 1 & $\mathrm{H}$ & $(E)-\mathrm{MeOCH}{ }_{2} \mathrm{CH}=\mathrm{CH}$ & 5 & 90 \\
2 & $4-\mathrm{OMe}$ & $(E)-\mathrm{PhCH}=\mathrm{CH}$ & 7 & 90 \\
3 & $4-\mathrm{Me}$ & $(Z)-\mathrm{PhCH}=\mathrm{CH}$ & 7 & 87 \\
4 & $4-\mathrm{OMe}$ & $(\mathrm{E})-\mathrm{BuCH}=\mathrm{CH}$ & 5 & 88 \\
5 & $4-\mathrm{NO}_{2}$ & $(Z)-\mathrm{PhCH}=\mathrm{CH}$ & 5 & 89 \\
6 & $4-\mathrm{NO}_{2}$ & $(Z)-\mathrm{BuCH}=\mathrm{CH}$ & 5 & 86 \\
\hline
\end{tabular}

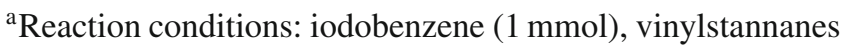
(1.2 mmol), Pd(0)-EGCG-CF (2.0 mol\%), DMF (3 mL), aerobic conditions, $60^{\circ} \mathrm{C}$

b'Isolated yield

turnover of Pd(0)-EGCG-CF under operating condition. The reusability of the catalysts is one of the most important benefits and makes them useful for commercial applications.

\section{Conclusion}

In conclusion, we have developed a simple and highly efficient method for the heterogeneous Stille coupling reaction of alkynylstannane with aryl iodides under aerobic conditions using $\mathrm{Pd}(0)$-EGCG-CF as a reusable catalyst. The significant advantages of this methodology are high yields, simple work-up procedure, excellent performance and reusability of the catalyst. The catalyst was separated from the reaction mixture and reused five times without significant loss of catalytic activity. This catalyst also has shown excellent activity on an industrial scale and in most cases it can be recovered from reaction mixture and reused. This methodology may be widely applied for the Stille reaction coupling in organic synthesis.

\section{Acknowledgements}

Authors thank the Ahar and Urmia Branches, Islamic Azad University, for partial financial support to carry out this research work.

\section{References}

1. (a) Stille J K 1986 Angew. Chem., Int. Ed. 25 508; (b) Diederich F and Stang P J 1998 Metal-catalyzed cross-coupling reactions (Weinheim: Wiley-VCH); (c) Shirakawa E and Hiyama T 1999 J. Organomet. Chem. 576 169; (d) Miyaura N 2002 Cross-coupling reaction (Berlin: Springer); (e) Hegedus L S 2002 In Organometallics in synthesis (ed) M Schlosser (Chichester: Wiley) p. 1123; (f) 2002 Handbook of organopalladium chemistry for organic synthesis (ed) 
E Negishi (New York: Wiley-Interscience); (g) Littke A F and Fu G C 2002 Angew. Chem., Int. Ed 41 4176; (h) Kamali T A, Habibi D and Nasrollahzadeh M 2009 Synlett 2601; (i) Kamali T A, Bakherad M, Nasrollahzadeh M, Farhangi S and Habibi D 2009 Tetrahedron Lett. 50 5459; (j) Stanforth S P 1998 Tetrahedron 54 263; (k) Kotha S, Lahiri K and Kashinath D 2002 Tetrahedron 589633

2. (a) Littke A F, Schwarz L and Fu G C 2002 J. Am. Chem. Soc. 124 6343; (b) Kim Y M and Yu S 2003 J. Am. Chem. Soc. 125 1696; (c) Menzel K and Fu G C 2003 J. Am. Chem. Soc. 125 3718; (d) Kim W S, Kim H J and Cho C G 2003 J. Am. Chem. Soc. 125 14288; (e) Dubbaka S R and Vogel P 2003 J. Am. Chem. Soc. 125 15292; (f) Wolf C and Lerebours R 2003 J. Org. Chem. 68 7077; (g) Wolf C and Lerebours R 2003 J. Org. Chem. 68 7551; (h) Mazzola R D Jr, Giese S, Benson C and West F G 2004 J. Org. Chem. 69 220; (i) Högenauer K 2001 Synlett 878; (j) Scrivanti A, Matteoli U, Beghetto V, Antonaroli S and Crociani B 2002 Tetrahedron 58 6881; (k) Kim N, Kwon M S, Park C M and Park J 2004 Tetrahedron Lett. 457057

3. (a) Herrmann W A, Öfele K, Preysing D v and Schneider S K 2003 J. Organomet. Chem. 687 229; (b) Hillier A C, Grasa G A, Viciu M S, Lee H M, Yang C and Nolan S P 2002 J. Organomet. Chem. 65369

4. (a) Alonso D A, Nájera C and Pacheco M C 2000 Org. Lett. 2 1823; (b) Chouday B M, Madhi S, Chowdari N S, Kantam M L and Sreedhar B 2002 J. Am. Chem. Soc. 124 14127; (c) Amatore C, Bahsoun A A, Jutand A, Meyer G, Ntepe A N and Ricard L $2003 \mathrm{~J}$. Am. Chem. Soc. 125 4212; (d) Miniére S and Cintrat J C 2001 J. Org. Chem. 667385

5. van Asselt R and Elsevier C J 1994 Tetrahedron 50 323

6. (a) Clapham B, Reger T S and Janda K D 2001 Tetrahedron 57 4637; (b) Schwarz J, Bohm V P W, Gardiner M G, Grosche M, Herrmann W A, Hieringer W and Raudaschl-Sieber G 2000 Chem. Eur. J. 61773

7. (a) Modarresi-Alam A R, Nasrollahzadeh M and Khamooshi F 2007 Arkivoc (xvi) 234; (b) Mohammad B, Hosseini Jamkarani S M, Kamali T A, Nasrollahzadeh $M$ and Mohajeri A 2010 Turk. J. Chem. 34 613; (c) Modarresi-Alam A R, Nasrollahzadeh M and Khamooshi F 2008 Sci. Iran.
15 452; (d) Modarresi-Alam A R, Khamooshi F, Nasrollahzadeh M and Amirazizi H A 2007 Tetrahedron 63 8723; (e) Habibi D and Nasrollahzadeh M 2012 Monatsh Chem. 143 925; (f) Nasrollahzadeh M, Bayat Y, Habibi D and Moshaee S 2009 Tetrahedron Lett. 50 4435; (g) Habibi D and Nasrollahzadeh M 2010 Synth. Commun. 40 3159; (h) Nasrollahzadeh M, Habibi D, Shahkarami Z and Bayat Y 2009 Tetrahedron 66 3866; (i) Habibi D, Nasrollahzadeh M and Kamali T A 2011 Green Chem. 13 3499; (j) Habibi D, Nasrollahzadeh M and Bayat Y 2011 Synth. Commun. 41 2135; (k) Habibi D and Nasrollahzadeh M 2012 Synth. Commun. 42 2023; (1) Habibi D, Heydari S and Nasrollahzadeh M 2012 J. Chem. Res. 36 573; (m) Habibi D, Nasrollahzadeh M, Mehrabi L and Mostafaee S 2012 Monatsh Chem. DOI 10.1007/s00706-012-0871-9; (n) Habibi D, Nabavi H, Nasrollahzadeh M 2013 J. Chem. Article ID 645313; (o) Bahari S, Mohammadi-Aghdam B, Molaei R, Gharibi Z 2012 Can. J. Chem. 90 784; (p) Bahari S, Mohammadi-Aghdam B, Sajadi S M and Zeidali F 2012 Bull. Korean Chem. Soc. 332251

8. (a) Müller W E G 2003 Integrated Computational Biology 43 3; (b) Smith J W 1968 Nature (London) 219 157; (c) Covington A D 1997 Chem. Soc. Rev 26 111; (d) Tang R X, Liao P, Liu X and Shi B 2005 Chem. Commun. 5882; (e) Wu H, Zhuo L, He Q, Liao X and Shi B 2009 Appl. Catal. A: Gen. 36644

9. (a) Liang B, Dai M, Chen J and Yang Z 2005 J. Org. Chem. 70 391; (b) Zhao H, Wang Y, Sha J, Sheng S and Cai M 2008 Tetrahedron 64 7517; (c) Carpino L A and Chen H-W 1979 J. Am. Chem. Soc. 101 390; (d) Huggins J M and Bergman R G $1981 \mathrm{~J}$. Am. Chem. Soc 103 3002; (e) Paquett L A and Wittenbrook L S 1967 J. Am. Chem. Soc. 89 4483; (f) Arcadi A, Cacchi S, Fabrizi G, Marinelli F and Pace P 1999 Eur. J. Org. Chem. 12 3305; (g) Hunter D H and Cram D J 1966 J. Am. Chem. Soc. 88 5765; (h) Chow H-F, Wan C-W, Low K-H and Yeung Y-Y 2001 J. Org. Chem. 66 1910; (i) Crisp G T, Turner P D and Stephens K A 1998 J. Organomet. Chem. 570 219; (j) Katritzky A R, Wang J, Karodia N and Li J 1997 J. Org. Chem. 62 4142; (k) Cheng J, Sum Y, Wang F, Guo M, Xu J H, Pan Y and Zhang Z 2004 J. Org. Chem. 69 5428; (1) Cai M, Zhou Z and Jiang J 2006 Eur. J. Org. Chem. 1400 